\title{
Cost-effectiveness Analysis of Cervical Cancer Screening Strategies Based on the Papanicolaou Smear Test in Korea
}

\author{
Min Jung Ko ${ }^{1 \&}$, Jimin Kim ${ }^{1 \&}$, Younhee Kim², Yoon Jae Lee ${ }^{3}$, Sung Ran Hong ${ }^{4}$, \\ Jae Kwan Lee ${ }^{5 *}$
}

\begin{abstract}
Background: Despite the increasing number of screening examinations performed for cervical cancer utilizing the Papanicolaou smear test (Pap test), few studies have examined whether this strategy is cost-effective in Korea. Objective: This study was conducted to evaluate the cost-effectiveness of cervical cancer screening strategies incorporating the Pap test based on age at the start and end of screening as well as screening interval. Materials and Methods: We designed four alternative screening strategies based on patient age when screening was started (20 or 30 years) and discontinued (lifetime, 79 years). Each strategy was assessed at screening intervals of 1, 2,3 , or 5 years. A Markov model was developed to determine the cost-effectiveness of the 16 possible cervical cancer screening strategies, and this was evaluated from a societal perspective. The main outcome measures were average lifetime cost, incremental quality-adjusted life-years (QALYs), and incremental cost-effectiveness ratio (ICER). Results: Compared with various strategies comprising younger starting age, discontinuation age, and longer screening intervals, strategies employing annual screening for cervical cancer starting at a target age of 30 years and above were the most cost-effective, with an ICER of 21,012.98 dollars per QALY gained (with a Korean threshold of 30,000,000 KRW or US\$27,272). Conclusions: We found that annual screening for cervical cancer beginning at a target age of $\mathbf{3 0}$ years and above is most cost-effective screening strategy. Considering the potential economic advantages, more intense screening policies for cervical cancer might be favorable among countries with high rates of cervical cancer and relatively low screening costs.
\end{abstract}

Keywords: Cervical cancer - cancer screening - Pap smear test - HPV infection - economic evaluation

Asian Pac J Cancer Prev, 16 (6), 2317-2322

\section{Introduction}

The benefits of screening for cervical cancer based on Papanicolaou smear test (Pap test) are well established; routine Pap testing has led to the decline in the incidence and mortality rate of cervical cancer (Canadian Task Force on Preventive Health Care, 2013; Sriplung et al., 2014). Indeed, after implementing a Pap test-based screening program in Korea, the age-adjusted incidence rate of cervical cancer per 100,000 women decreased noticeably from 16.3 in 1999 to 9.5 in 2012 (International Agency for Research on Cancer and World Health Organization, 2014). However, cervical cancer continues to rank as the sixth most common cancer in Korea (Jung et al., 2014), with an incidence 1.4 times higher than that of the USA in 2012 (International Agency for Research on Cancer and World Health Organization, 2014; Fernandez et al., 2014) despite a $17.58 \%$ increase in the number of screening examinations performed during the previous
6 years. Therefore, Korea now faces the challenge of implementing a screening program for cervical cancer to achieve increased effectiveness in terms of economics and clinical efficiency.

Korea implemented a biannual national screening program in 1999 targeting women aged 30 years and older for cervical cancer. The costs per case of screening and incidence of cervical cancer have a significant impact on the program's economic feasibility. The majority of previous economic studies on this screening program have focused on the relative cost-effectiveness of Pap tests compared with other tests, mainly the human papillomavirus (HPV) DNA test, at different screening intervals (Voko et al., 2012; Nahvijou et al., 2014). However, the costs associated with the HPV DNA test in Korea are 7 times greater than that of Pap test. Therefore, it would be preferable to identify effective screening strategies that utilize the Pap test rather than comparing screening test methods (de Kok et al., 2012). In addition,

${ }^{1}$ Division for Healthcare Technology Assessment Research, National Evidence-based Healthcare Collaborating Agency, ${ }^{2}$ Institute of Health and Environment and School of Public Health, Seoul National University, ${ }^{4}$ Department of Pathology, Cheil General Hospital \& Women's Healthcare Center, Catholic Kwandong University, ${ }^{5}$ Department of Gynecology, Korea University Guro Hospital, Seoul, ${ }^{3}$ Department of Oriental Gynecology, CHA Bundang Medical Center, CHA University, Seongnam, Republic of Korea ${ }^{\&}$ Equal contributors*For correspondence: jklee38@korea.ac.kr, jklee38@gmail.com 
only a few studies have performed cost-benefit analyses with respect to the age at which screening is started and discontinued, and the intervals at which screening incorporating the Pap test is performed (Moyer et al., 2012).

Therefore, the purpose of this study was to evaluate the cost-effectiveness of cervical cancer screening strategies based on the age at which screening is started and discontinued as well as the screening interval for Pap-based testing.

\section{Materials and Methods}

\section{Screening alternatives}

We designed four alternative screening strategies depending on the age at which screening was started (20 or 30 years old) and discontinued (life-time, 79 years old). Each alternative was assessed at 1-, 2-, 3-, and 5-year screening intervals. A summary of the corresponding 16 strategies is shown in Table 1.

The study protocol was approved by the ethics review committee of the National Evidence-based Healthcare Collaborating Agency, Seoul, Republic of Korea.

\section{Economic Evaluation}

A cohort simulation Markov model was developed to determine the cost-effectiveness of the 16 alternative cervical cancer screening strategies from a societal perspective in terms of cost and health outcomes. All analyses were conducted using Microsoft Excel 2007 and TreeAge Pro Suite (TREEAGE Software Inc.; Williamstown, MA, USA).

\section{Model Structure}

A Markov model with a one-year cycle was developed using TreeAge pro. The natural history of cervical cancer was subdivided into the following five sequential stages based on Kim et al. (2007): well, HPV infection, cervical intraepithelial neoplasia 1 (CIN1), CIN2/3, and cervical

Table 1. Overview of Screening Strategies for Cervical Cancer Using the Papanicolaou Smear Test

\begin{tabular}{|c|c|c|c|c|}
\hline No. & $\begin{array}{l}\text { Pap test strategy } \\
\text { (interval, starting } \\
\text { age, disconti- } \\
\text { nuation age) }\end{array}$ & Interval & $\begin{array}{l}\text { Starting age } \\
\text { (years old) }\end{array}$ & $\begin{array}{c}\text { Discontinua- } \\
\text { tionage } \\
\text { (years old) }\end{array}$ \\
\hline 1 & 1, 20- lifetime & \multirow[t]{4}{*}{1 year } & \multirow[t]{2}{*}{20} & Lifetime \\
\hline 2 & $1,20-79$ & & & 79 \\
\hline 3 & 1,30- lifetime & & \multirow[t]{2}{*}{30} & Lifetime \\
\hline 4 & $1,30-79$ & & & 79 \\
\hline 5 & 2,20 - lifetime & \multirow[t]{4}{*}{2 years } & \multirow[t]{2}{*}{20} & Lifetime \\
\hline 6 & $2,20-79$ & & & 79 \\
\hline 7 & 2,30 - lifetime & & \multirow[t]{2}{*}{30} & Lifetime \\
\hline 8 & $2,30-79$ & & & 79 \\
\hline 9 & 3,20 - lifetime & \multirow[t]{4}{*}{3 years } & \multirow[t]{2}{*}{20} & Lifetime \\
\hline 10 & $3,20-79$ & & & 79 \\
\hline 11 & 3, 30- lifetime & & \multirow[t]{2}{*}{30} & Lifetime \\
\hline 12 & $3,30-79$ & & & 79 \\
\hline 13 & 5,20 - lifetime & \multirow[t]{4}{*}{5 years } & \multirow[t]{2}{*}{20} & Lifetime \\
\hline 14 & $5,20-79$ & & & 79 \\
\hline 15 & 5,30 - lifetime & & \multirow[t]{2}{*}{30} & Lifetime \\
\hline 16 & $5,30-79$ & & & 79 \\
\hline
\end{tabular}

cancer. The target cohort consisted of 20-year-old Korean women, and the course of naturally developed cervical cancers was reviewed and followed. We hypothesized that progression from well to HPV infection, HPV infection to CIN1, and CIN1 to CIN2/3 would appear at distinct intervals, which has been noted previously for progression from HPV infection to CIN1 and CIN1 to CIN2/3 (Kim et al., 2007). In our model, individuals progressed from an infected state to the next level at an age-specific rate. Likewise, individuals also progressed from CIN2/3 to invasive cancer at a specific rate, and their cancer did not regress. We simulated the natural history of HPVinduced cervical dysplasia and incorporated standard procedures used in the screening, diagnosis, and treatment of precancerous lesions of the cervix.

\section{Model inputs}

All modeling assumptions and their sources are shown in Tables 2, 3, and 4.

Most of the input parameters were taken from the report by Kim et al. (2013). Korean data on the incidence of HPV infection and age-specific HPV incidence rates were derived from Shin et al. (2010). We estimated the rate of HPV infection by calibrating the age-specific HPV prevalence, frequency of sexual activity, and HPV infection-regression rate. We used health claims data from the National Health Insurance Service (NHIS) to estimate the probabilities of transitioning from CIN1 to CIN2/3 and from CIN2/3 to cervical cancer. The International Federation of Gynecology and Obstetrics (FIGO) annual report (Quinn et al, 2006) and data obtained from a review of previous studies were used to estimate the death rate for each health state. Furthermore, although information on Korea-specific cervical cancer mortality was available, it did not differentiate the increased risk in mortality as a function of increased severity of cervical cancer (i.e., stage 1 cervical cancer vs. stage 4 cervical cancer). Therefore, we employed the mortality rates of cervical cancer by stage reported by Lee et al. (2007), which we recalibrated to provide stage-specific cervical cancer mortality rates. Lastly, we used the national cancer screening rate reported by the NHIS to estimate the rate of screening.

\section{Diagnostic accuracy}

To ascertain the accuracy of cervical cancer screening methods in Korea, we performed a systematic review and meta-analysis for cervical cancer screening among asymptomatic women. We extracted sensitivity and specificity for the detection of CIN1 and CIN2/3, with colposcopy and histology reference standards. The sensitivity and specificity of CIN1 were 0.77 and 0.86 , while those of CIN2/3 were 0.97 and 0.73 , respectively.

Costs

All costs were converted to 2012 US dollars (\$) and discounted at a rate of $5 \%$. In addition to actual medical costs, we took into account societal perspectives such as costs for patient time, caregivers, and transportation. Screening costs were set as the NHIS medical fee. Physician, outpatient, and inpatient costs for other diagnostic procedures and treatments were extracted from 
Cost-effectiveness Analysis of Cervical Cancer Screening Strategies Based on the Papanicolaou Smear Test in Korea

NHIS claims data. We calculated the cost of screening for cervical cancer based on age-specific national cancerscreening rates and private cancer-screening rates. We computed the annual medical costs per patient diagnosed with CIN or cervical cancer from NHIS claims data, and estimated unofficial medical costs based on the results of patient surveys. We estimated the costs for transportation, time, and caregivers from NHI claims data, patient surveys, and unit costs reported by Kim et al. (Kim et al., 2013). The sum of these costs was 17,437.77, 6,297.08, and 3,416.97 for initial cancer state, follow-up 1 year after initial treatment, and follow-up 2, 3, and 4 years after

Table 2. Disease Probability Input Parameters

\begin{tabular}{|c|c|c|c|}
\hline Parameters & Probability & Source & Range \\
\hline \multicolumn{4}{|l|}{ Progression probability } \\
\hline Well history $\rightarrow$ HPV infection (age $[y]$ ) & & Calibration & $0.000-0.260$ \\
\hline Well $\rightarrow$ HPV infection (age [y]) & & Calibration & $0.000-0.260$ \\
\hline HPV infection $\rightarrow$ CIN1 & 0.025 & Debichi (2008) & \\
\hline HPV undetected $\rightarrow$ CIN1 & 0.025 & Debichi (2008) & \\
\hline $\mathrm{CIN} 1 \rightarrow \mathrm{CIN} 2 / 3$ treatment & 0.083 & $\operatorname{Kim}(2013)$ & \\
\hline CIN1 undetected $\rightarrow$ CIN2/3 treatment & 0.13 & $\operatorname{Kim}(2013)$ & \\
\hline $\mathrm{CIN} 2 / 3 \rightarrow$ Initial cervical cancer stage treatment & 0.0174 & $\operatorname{Kim}(2013)$ & \\
\hline CIN2/3 undetected $\rightarrow$ Initial cervical cancer stage treatment & 0.13 & Assumption & \\
\hline Initial cervical Cx undetected $\rightarrow$ symptoms & 0.15 & $\operatorname{Kim}(2012)$ & \\
\hline Initial cervical Cx $\rightarrow$ Cx 1-yr follow-up (age [y]) & & Kim (2012) & $0.701-0.877$ \\
\hline Cx 1-yr follow-up $\rightarrow$ Cx 2-yr follow-up (age [y]) & & $\operatorname{Kim}(2012)$ & $0.761-0.931$ \\
\hline Cx 2-yr follow-up $\rightarrow$ Cx 3-yr follow-up (age [y]) & & $\operatorname{Kim}(2012)$ & $0.813-0.980$ \\
\hline Cx 3-yr follow-up $\rightarrow$ Cx 4-yr follow-up (age [y]) & & $\operatorname{Kim}(2012)$ & $0.813-0.980$ \\
\hline Cx 4-yr follow-up $\rightarrow$ Cx cured (age [y]) & & $\operatorname{Kim}(2012)$ & $0.813-0.980$ \\
\hline Recurrent/Persistent $\mathrm{Cx} \rightarrow$ Recurrent/Persistent Cx 1-yr follow-up & 0.395 & Lee (2007) & \\
\hline $\begin{array}{l}\text { Recurrent/Persistent Cx 1-yr follow-up } \rightarrow \text { Recurrent/Persistent } \\
\text { Cx 2-yr follow-up (age [y]) }\end{array}$ & & FIGO report \& Lee (2007) & $0.813-0.980$ \\
\hline $\begin{array}{l}\text { Recurrent/Persistent Cx 2-yr follow-up } \rightarrow \text { Recurrent/Persistent } \\
\text { Cx 3-yr follow-up (age [y]) }\end{array}$ & & FIGO report \& Lee (2007) & $0.813-0.980$ \\
\hline $\begin{array}{l}\text { Recurrent/Persistent Cx 3-yr follow-up } \rightarrow \text { Recurrent/Persistent } \\
\text { Cx 4-yr follow-up (age [y]) }\end{array}$ & & FIGO report \& Lee (2007) & $0.813-0.980$ \\
\hline Recurrent/Persistent Cx 4-yr follow-up $\rightarrow$ Post Cx state (age [y]) & & FIGO report \& Lee (2007) & $0.813-0.980$ \\
\hline \multicolumn{4}{|l|}{ Regression probability } \\
\hline HPV infection $\rightarrow$ well (age [y]) & & Calibration & $0.670-0.752$ \\
\hline CIN1 $\rightarrow$ HPV infection or well & 0.45 & Debichi (2008) & \\
\hline CIN2/3 $\rightarrow$ CIN1 or well & 0.23 & Debichi (2008) & \\
\hline \multicolumn{4}{|l|}{ Proportion } \\
\hline Proportion of CIN1_well & 0.1 & Assumption & \\
\hline Proportion of CIN2/3_well & 0.5 & Assumption & \\
\hline
\end{tabular}

*HPV, human papillomavirus; CIN, cervical intraepithelial neoplasia; $\mathrm{Cx}$, invasive cervical cancer; FIGO report, The International Federation of Gynecology and Obstetrics (FIGO) annual report

Table 3. Cost Input Parameters

\begin{tabular}{|c|c|c|c|c|c|}
\hline Parameter & Total costs & $\begin{array}{l}\text { Formal medical } \\
\text { costs }\end{array}$ & $\begin{array}{l}\text { Informal medical } \\
\text { costs }\end{array}$ & $\begin{array}{c}\text { Caregiver, } \\
\text { transportation, } \\
\text { patient time costs }\end{array}$ & Source \\
\hline CIN1 & $2,521.70$ & 227.25 & $2,186.36$ & 108.09 & \multirow{10}{*}{$\begin{array}{l}\text { Kim et al. } \\
\text { (2013) }\end{array}$} \\
\hline Undetected CIN1 & 107.63 & 0 & 0 & 107.63 & \\
\hline $\mathrm{CIN} 2 / 3$ & $5,000.67$ & 735.16 & $3,781.05$ & 484.45 & \\
\hline Undetected CIN2/3 & 417.6 & 0 & 0 & 417.6 & \\
\hline Initial cancer & $17,437.77$ & $7,875.80$ & $5,655.68$ & $3,906.28$ & \\
\hline Follow up 1 yr after initial treatment & $6,297.08$ & $1,209.54$ & $4,511.83$ & 575.71 & \\
\hline $\begin{array}{l}\text { Follow up } 2,3 \text {, and } 4 \mathrm{yr} \text { after initial } \\
\text { treatment }\end{array}$ & $3,416.97$ & $1,005.04$ & $2,019.55$ & 392.38 & \\
\hline Recurrent/persistent cancer & $28,301.58$ & $19,431.92$ & $1,126.71$ & $7,742.95$ & \\
\hline $\begin{array}{l}\text { Follow up } 1 \text { yr after recurrent/ } \\
\text { persistent cancer }\end{array}$ & $4,248.22$ & $2,985.73$ & 96.99 & $1,165.49$ & \\
\hline $\begin{array}{l}\text { Follow up } 2,3 \text {, and } 4 \text { yr after } \\
\text { recurrent/persistent cancer }\end{array}$ & $4,967.41$ & $2,985.73$ & 818.22 & $1,163.45$ & \\
\hline Screening fee & 28.42 & 6.14 & 0 & 22.28 & \multirow{3}{*}{$\begin{array}{l}\text { NHIS Claims } \\
\text { data (2012) }\end{array}$} \\
\hline Pap test & 6.68 & 6.68 & 0 & 0 & \\
\hline Colposcopy test & 40.67 & 40.67 & 0 & 0 & \\
\hline
\end{tabular}


treatment, respectively.

\section{Outcomes}

Quality-adjusted life years (QALYs) were used as the primary measurement for assessing cost-effectiveness between alternative screening/testing algorithms (Table 4).

\section{Cost-effectiveness analysis}

The main outcome measures of the cost-effectiveness analysis were average lifetime cost, incremental QALYs, and incremental cost-effectiveness ratios (ICERs). We defined dominant screening strategies as those that offered more effective clinical outcomes (i.e., extended life expectancy) and cost less or had a more attractive cost per QALY than the next best option. The option of cervical cancer screening was dominated through extended dominance by other screening modalities. Differentiation reflected the fact that early detection and treatment of precancerous and cancer lesions alleviated some morbidity unaccounted for in the life years gained (LYG) measure.

\section{Sensitivity analysis}

Sensitivity analysis was conducted to reflect the uncertainty of variables. To better understand the impact on the results for different levels of participants along with the purpose of determining the validity of model results, sensitivity analysis was performed for Pap test accuracy, transition probability, and discount rate. We applied the lower and upper limits of the $95 \%$ confidence interval of mean accuracy, which was drawn from Kim et al.'s review of the literature (Kim et al., 2013). To consider differences in transition probability of CIN2/3 into cervical cancer, we applied a range of 50\% and 1.74\% (Moyer et al., 2012). In addition, we varied the assumptions for discount rates between $0 \%, 3.5 \%$, and $7 \%$.

\section{Results}

\section{Economic evaluation}

Cost-effectiveness results are shown in Table 5, which describe the lifetime costs and clinical benefits for different screening intervals. The strategy utilizing fiveyear intervals with target ages of 30 to 79 years old was the least expensive, but QALY was also reduced among the 16 strategies. Although the best QALY strategy was the one performed annually with a target age of 30 years and older, the cost was relatively substantial. Cost-effectiveness was dependent on the decision-makers' choice as to whether additional health gain was worth the additional cost (e.g., US\$27,272 per additional QALY gained). Under the Korean threshold of US\$27,272, the ICER for annual PAP screening starting at 30 years of age was US $\$ 21,012.98$, which was the most cost-effective. The next best was a biannual Pap test starting at 30 years of age, which had a cost of US $\$ 13,258.43$ per QALY.

\section{Sensitivity analysis}

We evaluated the robustness of our findings with sensitivity analyses. The magnitude of the incremental cost-effectiveness ratios remained unchanged when test accuracy was varied over a wide range of transition probabilities instead of previously reported data.

Figure 1 shows the dependence of cost-effectiveness on discount rate. Decreasing the discount rate from $7 \%$ to $0 \%$ while also increasing starting age considerably lowered the ICER. For a discount rate of $7 \%$, screening at two-year intervals with a target age of 30 years and above, which is the current Korean guideline, was the most costeffective strategy. Figure 1 also shows the variability of results according to PAP test accuracy. For the lower limit of test accuracy, the most cost-effective strategy consisted of two-year screening intervals with a target age of 20 to 79 years. On the other hand, for the upper limit of test accuracy, the most cost-effective strategy consisted of twoyear screening intervals with a target age of 20 years and above. After applying the transition probability of CIN $2 / 3$ into cervical cancer, which ranged from $1.74 \%$ to $50 \%$, the best strategy consisted of 1-year interval screening with a target age range of 20 to 79 years.

\section{Table 4. Utility Input Parameters}

\begin{tabular}{llc}
\hline Parameters & Probability & Source \\
\hline Well history state (age [y]) & $0.753-0.975$ & Kim et al. \\
Well state (age [y]) & $0.753-0.975$ & $(2013)$ \\
HPV infection (age [y]) & $0.753-0.975$ & \\
HPV infection undetected & $0.753-0.975$ & \\
(age [y]) & & \\
CIN1 & 0.937 & \\
CIN1 undetected (age [y]) & $0.753-0.975$ & \\
CIN2/3 & 0.933 & \\
CIN2/3 undetected (age [y]) & $0.753-0.975$ & \\
Initial cancer & 0.835 & \\
Follow up 1,2,3, and 4 yr & 0.898 & \\
after initial treatment & & \\
Post cancer cure (age [y]) & $0.753-0.975$ & \\
Recurrent/persistent cancer & 0.778 & \\
Follow up 1 yr after recurrent/ & 0.805 & \\
persistent cancer & & \\
Follow up 2,3, and 4 yr after & 0.778 & \\
recurrent/persistent cancer & & \\
\hline
\end{tabular}

Table 5. Cost-effectiveness Results of Cervical Cancer Screening Strategies

\begin{tabular}{llll}
\hline $\begin{array}{l}\text { Strategy (Interval, } \\
\text { Target age) }\end{array}$ & Cost & QALY & ICER \\
\hline PAP $(5,30-79)$ & 223.23 & 17.950253 & - \\
PAP $(5,20$-lifetime) & 286.72 & 17.953058 & dominated \\
PAP $(3,30-79)$ & 285.08 & 17.95349 & dominated \\
PAP $(5,20-79)$ & 303.29 & 17.953834 & dominated \\
PAP $(5,30$-lifetime) & 223.27 & 17.953834 & 11.17 \\
PAP $(2,30-79)$ & 348.3 & 17.956353 & dominated \\
PAP $(3,20$-lifetime) & 399.37 & 17.958563 & dominated \\
PAP $(3,20-79)$ & 423.16 & 17.95953 & dominated \\
PAP $(3,30$-lifetime) & 285.1 & 17.95953 & $10,853.55$ \\
PAP $(1,30-79)$ & 507.15 & 17.961365 & dominated \\
PAP $(2,20$-lifetime) & 515.87 & 17.963248 & dominated \\
PAP $(2,20-79)$ & 545.14 & 17.9643 & dominated \\
PAP $(2,30$-lifetime) & 348.34 & 17.9643 & $13,258.43$ \\
PAP $(1,20$-lifetime) & 798.49 & 17.970889 & dominated \\
PAP $(1,20-79)$ & 835.78 & 17.971852 & dominated \\
PAP $(1,30$-lifetime) & 507.03 & 17.971852 & $21,012.98$ \\
\hline
\end{tabular}

*Pap test, Papanicolaou Smear Test; QALYs: quality-adjusted life-years; ICER: incremental cost-effectiveness ratios; Exchange rate: $\$ 1=1,100$ KRW 




Figure 1. Sensitivity Analysis

\section{Discussion}

This study revealed 1) screening for cervical cancer with a target age of 30 years and above was the most cost-effective strategy with an ICER of US $\$ 21,012.98$ per QALY gained; and 2) annual screening was the most cost-effective strategy.

Western countries have previously recommended that annual screening for cervical cancer should start at an early age (Canadian Task Force on Preventive Health Care, 2013). In more recent years, the target age range for cervical cancer screening has narrowed, starting after 25 or 30 years of age and ending around 65 or 70 years of age based on clinical and economic aspects as well as government support (Moyer et al., 2012; Canadian Task Force on Preventive Health Care, 2013; Lee at al., 2013). However, the Korean Society of Gynecologic Oncology currently recommends that annual screening be performed for women ages 20 to 70 years based on the high incidence of cervical cancer, easy access to screening, and low medical cost in Korea (Lee et al., 2013).

The results of this study suggest that screening starting at 30 years of age is the most cost-effective approach. Specifically, there is a limited health gain for initiating screening in women under the age of 30 owing to the relatively low incidence of cervical disease. Although the overall incidence of cervical cancer in Korea was 11.7 per 100,000 in 2011, the age specific incidence for 25-29 years of age was 6.5, which is half that of the 30-34 age group (Ministry for Health and Welfare, 2011). Consistent with our findings, recommendations based on high-quality evidence suggest that routine screening for cervical cancer be performed for women 30 years of age and above (Canadian Task Force on Preventive Health Care, 2013; Netherlands Health council, 2011). Conversely, other guidelines based on moderate or weak-quality evidence recommend that screening be started between 20 and 25 years of age, and be performed at intervals of two or three years (de Kok et al., 2012; Canadian Task Force on Preventive Health Care, 2013; Australian Government, 2014).

An important result of this study was that no screening strategy with a discontinuation age of 79 years was cost-effective. Most cervical cancer screening policies suggest a discontinuation age of 65 to 70 years, under the assumption of appropriate previous screening (London,
2011; de Kok et al., 2012; Australian Government ,2014). However, there appears to be no substantial evidence to support such discontinuation, since the majority of studies we reviewed excluded this age group (Mun et al., 2011; Canadian Task Force on Preventive Health Care, 2013). In addition, protective effects of screening have been identified in women aged 65 to 70 years and above (Andrae et al., 2008). Indeed, the current consensus statement developed by US National Institutes of Health Consensus Development Conference Statement on Cervical Cancer encourages regular screening for women ages 65 and older, which is contrary to US Preventive Service Task Force guidelines (2012) (Lee et al., 2012). Moreover, Lee et al. (2013) emphasized the necessity of screening for cervical cancer among Korean women 65 years of age and older, since the incidence was double that of women under the age of 65 .

With respect to the appropriate screening interval for cervical cancer, annual screening appears to be the most cost-effective strategy. Most western countries recommend an interval for cervical cancer screening of 3 or 5 years (de Kok et al., 2012; Canadian Task Force on Preventive Health Care, 2013; Lee et al., 2013). However, for countries with a high risk of cervical cancer and low screening costs, more intense guidelines may be beneficial. Specifically, such guidelines may recommend that it is necessary to begin screening at a younger age, end at an older age, and have a shorter interval between screenings (de Kok et al., 2012).

Overall, our results were susceptible to uncertainties of the model structure and input parameters, which is in line with other health economic models. Our results were also sensitive to the discount rate applied. Since the current investment will produce health benefits only after a long period of time, high discount rate devalues the benefits more than costs (Voko et al., 2012). When applying a transition probability of $1.74 \%$ rather than $50 \%$, our results were sensitive and the ICER increased considerably.

Our study also had several limitations. First, several input parameters of the model including HPV infection rate and certain transition probabilities were estimated by calibration, since there were no available Korean data. However, in estimating the HPV infection rate, we tried to minimize this limitation by considering relevant empirical factors such as HPV prevalence, frequency of sexual activity, and HPV infection regression rate. Secondly, our analysis of cost-effectiveness was based on Korean cost data that has limited applicability to other healthcare settings. In particular, the cost related to the Pap test screening is much lower in Korea compared to other countries. However, costs were obtained from representative national data, which may be generalizable among countries with similar healthcare costs. Moreover, we considered various costs associated with patient time, caregivers, and transportation, in addition to direct medical costs. Lastly, the results of this study were sensitive to discount rate, Pap test accuracy, and transition probability.

In conclusion, the results of our study suggest that strategies employing annual screening for cervical cancer with a target age of 30 years and above are the most costeffective in Korea. Considering the potential economic 
Min Jung Ko et al

advantages, more intense screening policies for cervical cancer may be favorable among countries with a high risk of cervical cancer and low screening costs.

\section{Acknowledgements}

This study was supported by the National Evidencebased Healthcare Collaborating Agency (NC13-009).

\section{References}

Andrae B, Kemetli L, Sparen P, et al (2008). Screeningpreventable cervical cancer risks: evidence from a nationwide audit in Sweden. J Natl Cancer Inst, 100, 622-9.

Australian Government, Department of Health and Ageing. National cervical screening program 2014. Available from: www.cancerscreening.gov.au/internet/screening/publishing. nsf/Content/cervical-about.

Canadian Task Force on Preventive Health Care, Dickinson J, Tsakonas E, et al (2013). Recommendations on screening for cervical cancer. CMAJ, 185, 35-45.

de Kok IM, van Rosmalen J, Dillner J, et al (2012). Primary screening for human papillomavirus compared with cytology screening for cervical cancer in European settings: cost effectiveness analysis based on a Dutch microsimulation model. $B M J, 344, \mathrm{e} 670$.

Fernandez ME, Savas LS, Lipizzi E, et al (2014). Cervical cancer control for Hispanic women in Texas: strategies from research and practice. Gynecol Oncol, 132, 26-32.

International Agency for Research on Cancer and World Health Organization. EUCAN (2014). Cancer incidence, mortality and prevalence. Available from: http://eco.iarc.fr/eucan/ CancerOne. asp $x$ ?Cancer $=25 \&$ Gender $=2$.

Jung KW, Won YJ, Kong HJ, et al (2014). Cancer statistics in Korea: incidence, mortality, survival, and prevalence in 2011. Cancer Res Treat, 46, 109-23.

Kim JJ,Andres-Beck B, Goldie SJ (2007). The value of including boys in an HPV vaccination programme: a cost-effectiveness analysis in a low-resource setting. Br J Cancer, 97, 1322-8.

Kim YH, Kim YJ, Kim J, et al (2013). Economic evaluation of HPV vaccination. National Evidence-based healthcare Collaborating Agency. NECA, Seoul.

Lee JK, Hong JH, Kang S, et al (2013). Practice guidelines for the early detection of cervical cancer in Korea: Korean society of gynecologic oncology and the Korean society for cytopathology 2012 edition. J Gynecol Oncol, 24, 186-203.

London (UK): The Service. National Health Service NHS cervical screening programme 2011.

Available from: www.cancerscreening.nhs.uk/cervical

Lee S, Park HT, Hong JH, et al (2013). Assessment of cervical cancer screening policy in Korea for women over age $65 . J$ Geriatr Oncol, 4, 382-7.

Lee SH, Lee Y, Kim HS, et al (2007). Analysis of clinical characteristics and prognosis in patient with recurrent cervical cancer. Korean J Obstet Gynecol, 51, 173-81.

Lee YH, Choi KS, Lee HY, Jun JK (2012). Current status of the National Cancer Screening Program for cervical cancer in Korea, 2009. J Gynecol Oncol, 23, 16-21.

Ministry for Health and Welfare. Annual report of cancer statistics in Korea in 2011. Available from: http://www. cancer.or.kr/

Moyer VA (2012). Screening for cervical cancer: U.S. Preventive Services Task Force recommendation statement. Ann Inter Med, 156, 880-91.

Mun JY, Han MA, Lee HY, et al (2011). Cervical cancer screening in Korea: report on the national cancer screening programme in 2008. Asian Pac J Cancer Prev, 12, 1961-4.

Nahvijou A, Hadji M, Marnani AB, et al (2014). A systematic review of economic aspects of cervical cancer screening strategies worldwide: discrepancy between economic analysis and policymaking. Asian Pac J Cancer Prev, 15, 8229-37.

Netherlands Health council. Population screening for cervical cancer (2011). Available from: www.gezondheidsraad.nl/en/ publications/population-screening-cervical-cancer.

Quinn MA, Benedet JL, Odicino F, et al (2006). Carcinoma of the cervix uteri. FIGO $26^{\text {th }}$ Annual report on the results of treatment in gynecological cancer. Int J Gynaecol Obstet, 95, 43-103.

Shin KR, Park HJ, Bae KE, et al (2012). Sexual behavior, health risk behaviors related to reproductive health, and sexual experiences among Korean college students. J Korean Acad Adult Nurs, 22, 614-33.

Sriplung H, Singkham P, Iamsirithaworn S, et al (2014). Success of a cervical cancer screening program: trends in incidence in Songkhla, southern Thailand, 1989-2010, and prediction of future incidences to 2030. Asian Pac J Cancer Prev, 15, 10003-8.

Voko Z, Nagyjanosi L, Margitai B, et al (2012). Modeling costeffectiveness of cervical cancer screening in Hungary. Value Health, 15, 39-45. 